CLINICAL STUDY

\title{
Retinoic acid for redifferentiation of thyroid cancer - does it hold its promise?
}

\author{
Thomas Grüning, Claudia Tiepolt, Klaus Zöphel, Jan Bredow, Joachim Kropp and Wolf-Gunter Franke \\ Department of Nuclear Medicine, Carl Gustav Carus Medical School, University of Dresden, Fetscherstrasse 74, 01307 Dresden, Germany
}

(Correspondence should be addressed to T Grüning, Department of Nuclear Medicine, Derriford Hospital, Plymouth, Devon PL6 8DH, UK; Email: Thomas.Gruning@phnt.swest.nhs.uk)

\begin{abstract}
Objectives: To evaluate the effectiveness of isotretinoin for improving ${ }^{131} \mathrm{I}$ uptake in recurrent/metastasized thyroid cancer with no/insufficient ${ }^{131}$ I uptake.

Design: Retrospective analysis of 25 patients treated between June 1999 and May 2001.

Methods: 15 female and 10 male patients were given isotretinoin at $1 \mathrm{mg} / \mathrm{kg}$ for 3 months, followed by

${ }^{131}$ I treatment. All patients received a ${ }^{131}$ I scan $72 \mathrm{~h}$ after administration, thyroglobulin measurement, chest X-ray and ultrasound of the neck, and some patients underwent a ${ }^{18}$ F-fluorodeoxyglucose (FDG) positron emission tomography $(n=14)$ and computed tomography scan of the chest $(n=11)$. Results: In two out of 14 patients with raised thyroglobulin but no ${ }^{131}$ I uptake, a slightly improved ${ }^{131}$ I uptake was seen. In a further 11 patients an improvement of ${ }^{131}$ I uptake of known lesions was desired or further non- ${ }^{131}$ I-accumulating lesions were known. A dosimetrically relevant improvement of uptake was seen in three of these patients. ${ }^{18}$ F-FDG uptake and thyroglobulin did not correlate with the success/failure of the isotretinoin treatment. Side effects including a strong 'sunburn', cheilitis, mucositis, conjunctivitis and raised transaminases occurred in two-thirds of patients. They were of an overall tolerable level and were reversible after isotretinoin had been stopped. Conclusion: From our clinical experience over a period of 2 years we conclude that the therapeutic effect of isotretinoin in thyroid cancer is certainly less than previously reported. An indiscriminate use of isotretinoin in all patients with otherwise untreatable thyroid cancer cannot be recommended.
\end{abstract}

European Journal of Endocrinology 148 395-402

\section{Introduction}

Differentiated thyroid cancer has in most cases a very favourable prognosis. There are three standard therapeutic modalities which have been used for many years: thyroidectomy, followed by radioiodine treatment courses to ablate remnant thyroid tissue and, if necessary, irradiate any metastases, and finally levothyroxine is given life-long to suppress the production of thyroidstimulating hormone (TSH).

About one-third of metastasized or recurrent thyroid carcinomas dedifferentiate over time, and this will eventually make them inaccessible to radioiodine therapy. For instance, a reduced expression of the thyroidal sodium/iodide symporter (NIS) may lead to a decreased iodine uptake and make radioiodine treatment ineffective (1). Loss of TSH receptor expression will take the growth-regulating effect of TSH away and will make levothyroxine treatment for growth suppression ineffective. For these reasons, there is a clinical need for new treatment options for those patients in whom established modalities are no longer effective $(2,3)$. It is hoped that retinoic acid will fulfil this need by both increasing radioiodine uptake to levels high enough to deliver a radiation dose sufficient for treatment and by exerting a direct anti-tumour effect.

Retinoic acid is a biologically active metabolite of vitamin A. Its most common clinical application is in severe acne vulgaris (4). In oncology, its main use so far is in acute promyelocytic leukaemia where up to $90 \%$ remission can be achieved (5-8).

Retinoic acid is known to affect a wide spectrum of genetic, proliferative and differentiation processes. At the molecular level, it mediates these effects by binding to retinoic acid receptors (RAR) and retinoid X receptors (RXR) which are transcription factors belonging to the nuclear receptor superfamily. RAR-RXR heterodimers modulate the transcription of target genes by binding to retinoic acid response elements (RARE) in their promoter (9). Retinoic acid receptors are also expressed in human thyroid carcinomas, although to a varying degree (10). In vitro studies have shown 
that treatment of thyroid cancer cells with retinoic acid leads to: (i) induction of the type I $5^{\prime}$-deiodinase (11, 12) which de-iodates thyroxine to tri-iodothyronine and whose activity is reduced in thyroid cancer compared with normal thyroid tissue (13), (ii) expression of NIS mRNA which may be decreased or even lost in thyroid cancer $(1,14,15)$, (iii) increased expression of thyroglobulin mRNA (16), (iv) induction of alkaline phosphatase which is a differentiation marker (12), (v) induction of the intercellular adhesion molecule-1 (ICAM-1) (17), (vi) a decreased expression of CD97, a dedifferentiation marker that is highly expressed in anaplastic thyroid carcinomas, whereas its expression in differentiated thyroid carcinomas is either lacking or low (18), and (vii) an antiproliferative effect (15).

These changes are seen as partial, rather than complete redifferentiation of thyroid cancer cells. Nevertheless, it is hoped that the partial restoration of thyrocyte-specific functions will be sufficient to make these patients accessible to established treatment modalities, especially radioiodine, again. This hope is supported by a small number of pilot studies. We started isotretinoin treatments shortly after the first encouraging results had been published. The study presented here will take a critical look back on the use of isotretinoin in a clinically relevant patient population over a period of 2 years.

\section{Materials and methods}

\section{Patients}

Between June 1999 and May 2001, 25 patients (15 female, 10 male, mean age 66 (range 37-86) years) were treated with isotretinoin prior to radioiodine therapy at the Department of Nuclear Medicine in Dresden. Details of each patient can be found in Table 1. In summary, there were 10 papillary, 13 follicular and 2 oncocytic carcinomas. Staging was as follows: T staging: $5 \mathrm{~T} 2,9 \mathrm{~T} 3,10 \mathrm{~T} 4,1 \mathrm{Tx}$; N staging: $6 \mathrm{NO}, 10 \mathrm{N1}, 9 \mathrm{Nx}$; and M staging: $3 \mathrm{MO}, 17 \mathrm{M1}, 5 \mathrm{Mx}$.

Previous treatment included thyroidectomy, on average 6 (range 1-39) years previously, followed by $5(1-15){ }^{131}$ I treatments with a cumulated activity of $19(3.8-54) \mathrm{GBq}$.

\section{Indications/inclusion criteria}

There were two distinct groups of patients in whom isotretinoin treatment was considered to be indicated. Patients in group $1(n=14)$ had a negative post-treatment radioiodine scan, but evidence of local recurrence/cervical lymph node metastases $(n=7)$, mediastinal lymph node/distant metastases $(n=3)$ or both local and distant disease $(n=4)$ from ultrasound, chest X-ray, computed tomography (CT) scan of the chest, bone scan or ${ }^{18}$ F-fluorodeoxyglucose (FDG) position emission tomography (PET). In group $2(n=11)$ post-treatment scans showed that ${ }^{131} \mathrm{I}$ uptake of known lesions had decreased over time although there was other evidence of disease progression and an improvement of uptake was intended or the patient had both non- ${ }^{131}$ I-accumulating and ${ }^{131}$ I-accumulating lesions. None of these patients had disease confined to the neck, but seven patients had mediastinal lymph node/distant metastases and four patients had both local recurrence/cervical lymph node metastases and mediastinal lymph node/distant metastases.

\section{Isotretinoin}

The dosing scheme was $1 \mathrm{mg} / \mathrm{kg}$ body weight given for 3 months prior to radioiodine treatment. This resulted in 40-100 mg Roaccutan given orally, i.e. $0.96(0.6-1.3) \mathrm{mg} / \mathrm{kg}$ for 85 (31-98) days.

\section{Radioiodine treatment and scan}

This was performed after standard thyroid hormone withdrawal. Patients were not advised to adhere to a low iodine diet since Dresden lies in an area of iodine deficiency. A mean activity of $4.4(3.2-7.8) \mathrm{GBq}{ }^{131} \mathrm{I}$ was administered orally with patients fasted for $2 \mathrm{~h}$ before and after administration.

A whole-body scan was performed $72 \mathrm{~h}$ after radioiodine administration. We used a double-head gamma camera with a large field-of-view (Genesys, ADAC Laboratories, Milpitas, CA, USA) and the following acquisition parameters: high-energy collimators, 20\% energy window, $512 \times 512 \times 16$ matrix, gantry speed $15 \mathrm{~cm} / \mathrm{min}$.

A partially shielded activity standard was placed between the calves of the patients to allow semiquantitative determination of the radioiodine uptake of particular lesions. This was performed using the geometric mean of the anterior and posterior images and the region-of-interest (ROI) technique with appropriate correction for background activity. A lesion was characterized by its uptake (expressed as a percentage of the administered activity) and its contrast (target-to-background ratio, expressed as the ratio of counts in the lesion vs those in a background ROI of equal size, usually positioned in the upper arm).

Each scan was evaluated alongside the previous scan by two experienced observers blinded to the use of retinoic acid who classified the scans independently as showing a clearly improved (i. e. potentially therapeutically relevant) uptake or minor improvement or no change.

\section{Other diagnostic tests}

Measurement of serum thyroglobulin (TG), including recovery, was performed using an immunoradiometric assay (SELco Tg, Medipan Diagnostica, Selchow, Germany) with a functional assay sensitivity of 
Table 1 Clinical details of patients included in this study and results of diagnostic tests performed. Thyroglobulin values in brackets were obtained when the patient was on levothyroxine and are, therefore, not directly comparable.

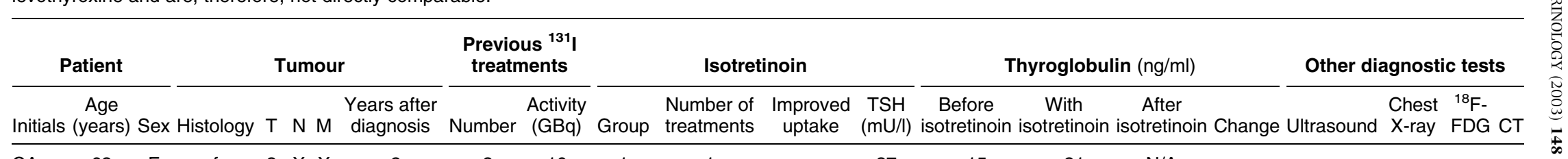

\begin{tabular}{|c|c|c|c|c|c|c|c|c|c|c|c|c|c|c|c|c|c|c|c|c|c|}
\hline CA & 63 & $\mathrm{~F}$ & $f$ & 3 & $x>$ & $X$ & 2 & 3 & 10 & 1 & 1 & + & 37 & 15 & 21 & $\mathrm{~N} / \mathrm{A}$ & + & + & - & - & \\
\hline LB & 62 & $M$ & fo & 2 & $x>$ & $X$ & 6 & 4 & 13 & 1 & 1 & 0 & 45 & 12 & 19 & (16) & + & + & - & + & \\
\hline $\mathrm{RB}$ & 64 & $\mathrm{~F}$ & $f$ & 2 & 0 & 1 & 10 & 12 & 44 & 2 & 4 & 0 & 31 & 4900 & 3300 & 4000 & - & + & - & & + \\
\hline ED & 77 & $\mathrm{~F}$ & $f$ & 3 & $1\rangle$ & $x$ & 7 & 5 & 19 & 1 & 1 & 0 & 5 & 300 & 1400 & (280) & + & + & - & & \\
\hline TE & 37 & $M$ & $p$ & 4 & 1 & 1 & 2 & 5 & 19 & 2 & 1 & 0 & 80 & 37 & 26 & $\mathrm{~N} / \mathrm{A}^{\prime}$ & - & & + & & - \\
\hline$R G$ & 76 & $M$ & $f$ & 2 & $\mathrm{X}$ & 1 & 2 & 5 & 19 & 2 & 2 & 0 & 46 & 200 & 160 & 150 & - & + & - & + & + \\
\hline$A G$ & 79 & $\mathrm{~F}$ & $f$ & 3 & X & 1 & 4 & 9 & 34 & 2 & 2 & + & 73 & 1100 & 3300 & 2300 & + & - & - & + & - \\
\hline UH & 76 & $\mathrm{~F}$ & $p$ & 4 & $1 b$ & 1 & 2 & 4 & 23 & 1 & 1 & 0 & 68 & 69 & 43 & (2) & $\S$ & - & - & + & \\
\hline $\mathrm{BH}$ & 63 & $\mathrm{~F}$ & $f$ & 4 & X & 1 & 1 & 1 & 4 & 2 & 2 & + & 58 & 5800 & 3000 & 2800 & - & - & + & & \\
\hline RK & 69 & $\mathrm{~F}$ & $f$ & 3 & X & 1 & 11 & 15 & 54 & 2 & 1 & + & 18 & 4600 & 5800 & (1300) & + & + & + & + & \\
\hline RK & 62 & $\mathrm{~F}$ & $f$ & 4 & 0 & 1 & 1 & 2 & 7 & 1 & 2 & 0 & 34 & 11 & 9 & $\mathrm{~N} / \mathrm{A}$ & 0 & - & + & & + \\
\hline JK & 49 & $M$ & $p$ & 4 & 1 & 0 & 1 & 2 & 8 & 1 & 1 & 0 & 74 & 17 & 183 & N/A & + & - & - & - & \\
\hline SM & 42 & $\mathrm{~F}$ & $f$ & $4 b$ & $1 b$ & 1 & 1 & 3 & 11 & 2 & 1 & 0 & 70 & 140 & 90 & (8) & - & - & - & - & + \\
\hline WM & 67 & $M$ & $f$ & 3 & 0 & 1 & 1 & 2 & 8 & 2 & 2 & 0 & 80 & 28 & 31 & 11 & 0 & - & - & & \\
\hline 10 & 62 & $\mathrm{~F}$ & $p$ & $x$ & $x$ & 1 & 38 & 10 & 34 & 1 & 1 & 0 & 19 & 11 & 43 & (16) & + & + & + & & + \\
\hline GR & 67 & $\mathrm{M}$ & fo & 3 & 0 & 1 & 10 & 10 & 46 & 2 & 2 & 0 & 51 & 4800 & 3600 & 3300 & - & - & - & & \\
\hline GR & 49 & $\mathrm{~F}$ & $f$ & 3 & $x>$ & $x$ & 4 & 3 & 10 & 1 & 1 & 0 & 21 & 1800 & 21 & N/A & - & - & - & + & \\
\hline HS & 69 & $M$ & pf & 4 & $1 b$ & 1 & 2 & 4 & 19 & 2 & 2 & 0 & 40 & 76 & 72 & 300 & 0 & - & + & & \\
\hline $\mathrm{HS}$ & 63 & $\mathrm{~F}$ & $p$ & 2 & $1 \mathrm{~b}$ & 1 & 1 & 2 & 7 & 1 & 1 & + & $\mathrm{N} / \mathrm{A}$ & 2 & 27 & (6) & + & + & + & + & + \\
\hline HS & 86 & $M$ & $p$ & 4 & 0 & 1 & 10 & 3 & 10 & 1 & 1 & 0 & 23 & N/A & 430 & $\mathrm{~N} / \mathrm{A}$ & $\S$ & & + & & \\
\hline ES & 86 & $\mathrm{~F}$ & $p$ & 4 & X & $x$ & 2 & 2 & 8 & 1 & 1 & 0 & 80 & 20 & 21 & $\mathrm{~N} / \mathrm{A}$ & 0 & - & - & & \\
\hline FS & 70 & M & $f$ & 3 & 1 & 0 & 7 & 3 & 10 & 1 & 1 & 0 & 60 & 290 & 720 & $\mathrm{~N} / \mathrm{A}$ & + & + & - & + & \\
\hline VT & 63 & $\mathrm{~F}$ & $f$ & 3 & 0 & 1 & 1 & 2 & 11 & 1 & 1 & 0 & 29 & 320 & 190 & (144) & - & - & - & + & - \\
\hline AT & 72 & $\mathrm{~F}$ & $p$ & 2 & 1 & 0 & 7 & 2 & 8 & 1 & 1 & 0 & 5 & 0 & 0 & 0 & 0 & + & - & + & - \\
\hline HU & 71 & $M$ & $p$ & 4 & 1 & 1 & 4 & 10 & 38 & 2 & 1 & 0 & 64 & 250 & 280 & (60) & 0 & - & + & & \\
\hline
\end{tabular}

$\S \mathrm{TG}$ not comparable due to widely different serum TSH levels; *values apply to first treatment where patients had more than one course of isotretinoin; N/A, not available; f, follicular; fo, oncocytic; p, papillary; pf, follicular variant of papillary carcinoma. 
$0.4 \mathrm{ng} / \mathrm{ml}$ (19). Anti-thyroglobulin antibodies were measured using a radioligand assay (CentAK anti-Tg, Medipan). These measurements were always performed when patients were off levothyroxine. A chest X-ray and ultrasound examination of the neck were routinely carried out in all patients. If clinically indicated, an ${ }^{18} \mathrm{~F}$ FDG PET scan $(n=14)$, CT scan of the chest without contrast medium $(n=11)$ or bone scan $(n=4)$ were performed.

\section{Results}

\section{Radioiodine uptake}

Group 1 Visual evaluation of ${ }^{131}$ I uptake showed a minor improvement in two out of 14 patients, and no change in the remaining 12 patients. In those two patients, ROI analysis (Table 2) revealed that the uptake of identifiable lesions increased in one patient (HS, Fig. 1) and did not increase in the other patient (CA). Contrast showed a mixed response in the former and was unchanged in the latter patient.

Group 2 In three out of 11 patients, improvement of radioiodine uptake was clearly visible. There was no change in the remaining patients. ROI analysis (Table 2) in those three patients showed (i) a good response in patient AG (Fig. 2) with both the uptake and contrast improved, (ii) a decreased uptake, but improved contrast in patient $\mathrm{RK}$, and (iii) conflicting results in patient $\mathrm{BH}$ with an improved uptake on visual evaluation and no significant change of both uptake and contrast in ROI analysis.

Further diagnostic tests Changes in serum TG levels $(>20 \%)$ were indeterminate: increase $(n=9)$, decrease $(n=8)$, no change $(n=6)$, and not comparable due to greatly different serum TSH levels $(n=2)$. TG levels and ${ }^{18}$ F-FDG uptake did not correlate with response of ${ }^{131}$ I uptake to isotretinoin treatment.

\section{Clinical response}

Further data and a clinical history of patients AG and HS are presented in Figs 1 and 2. The course of disease in the other three patients showing at least some response is described briefly here. In patient CA with a moderately well differentiated follicular carcinoma, only minimal radioiodine uptake in a local recurrence could be induced and this was not considered therapeutically significant. TG levels were $21 \mathrm{ng} / \mathrm{ml}$ at this time. Therefore, this patient was referred for surgery. Histology, however, did not show any evidence of malignancy. After 1 year of follow-up she was clinically asymptomatic, with ultrasound of the neck showing no residual disease. TG levels were $5 \mathrm{ng} / \mathrm{ml}$ under levothyroxine.

Patient BH had an invasive follicular, partially dedifferentiated carcinoma infiltrating cervical soft tissues and pulmonary metastases. After isotretinoin/radioiodine treatment, a partial remission of pulmonary metastases on the chest X-ray was shown. However, she developed a poorly differentiated local recurrence and lymph node metastases and received repeat surgery three months later. Another isotretinoin/radioiodine treatment was given, again with partial regression of the pulmonary metastases. Since the patient was clinically only mildly symptomatic, no further treatment was given.

Patient RK with a widespread metastasized follicular carcinoma (pulmonary, bone, liver, lymph nodes) had had 15 previous radioiodine treatments. However, radioiodine uptake had decreased over time. After isotretinoin pretreatment an improved radioiodine uptake in the known lesions could be achieved. The disease was initially stable on chest X-ray and ultrasound. However, TG levels were rising. Further radioiodine treatments could not be performed because of a profound thrombopenia. The patient was then admitted to another hospital with cardiopulmonary decompensation due to a malignant pleural effusion and ascites.

Table 2 Uptake $(\mathrm{U})$ and contrast (C) of lesions in those patients (CA, AG, BH, RK, HS) who showed an improvement on visual evaluation. All figures given are percentages related to the last radioiodine scan before isotretinoin was given (100\%). For further details of these patients, refer to Table 1 and Figs 1 and 2 .

\begin{tabular}{|c|c|c|c|c|c|c|c|c|c|c|c|c|c|c|}
\hline & \multirow{2}{*}{\multicolumn{2}{|c|}{$\begin{array}{c}\text { CA } \\
(\text { Feb 00 })^{\star} \\
\text { Dec } 00 \dagger\end{array}$}} & \multicolumn{4}{|c|}{$\begin{array}{c}\text { AG } \\
(\text { Apr 00)* }\end{array}$} & \multicolumn{4}{|c|}{$\begin{array}{c}\text { BH } \\
(\operatorname{Mar} 00)^{*}\end{array}$} & \multirow{2}{*}{\multicolumn{2}{|c|}{$\begin{array}{c}\begin{array}{c}\text { RK } \\
(\text { Mar 00)* }\end{array} \\
\text { Jun 00† }\end{array}$}} & \multirow{2}{*}{\multicolumn{2}{|c|}{$\begin{array}{c}\begin{array}{c}\text { HS } \\
(\text { Apr 99 }\end{array} \\
\text { Dec 99† }\end{array}$}} \\
\hline & & & \multicolumn{2}{|c|}{ Aug $00 \dagger$} & \multicolumn{2}{|c|}{ Jan $01 \dagger$} & \multicolumn{2}{|c|}{ Jul 00† } & \multicolumn{2}{|c|}{ Nov $00 \dagger$} & & & & \\
\hline & $U$ & C & $U$ & C & $\mathrm{U}$ & C & U & C & U & C & $\mathrm{U}$ & C & $\mathrm{U}$ & C \\
\hline$R$ lung & 44 & 94 & 143 & 137 & 152 & 117 & 106 & 100 & 117 & 92 & 47 & 134 & 175 & 106 \\
\hline L lung & 42 & 90 & 129 & 125 & 141 & 110 & 109 & 101 & 115 & 91 & 53 & 148 & 157 & 98 \\
\hline Thyroid bed & 38 & 88 & & & & & 116 & 108 & 109 & 85 & 83 & 232 & & \\
\hline Mediastinum & & & 179 & 175 & 292 & 226 & & & & & 39 & 108 & 178 & 129 \\
\hline Upper R lung & & & & & & & & & & & 61 & 160 & & \\
\hline $\mathrm{R}$ humerus & & & & & & & & & & & 42 & 117 & & \\
\hline
\end{tabular}

*, pre-isotretinoin; $\dagger$, on isotretinoin.

$R$, right; L, left. 


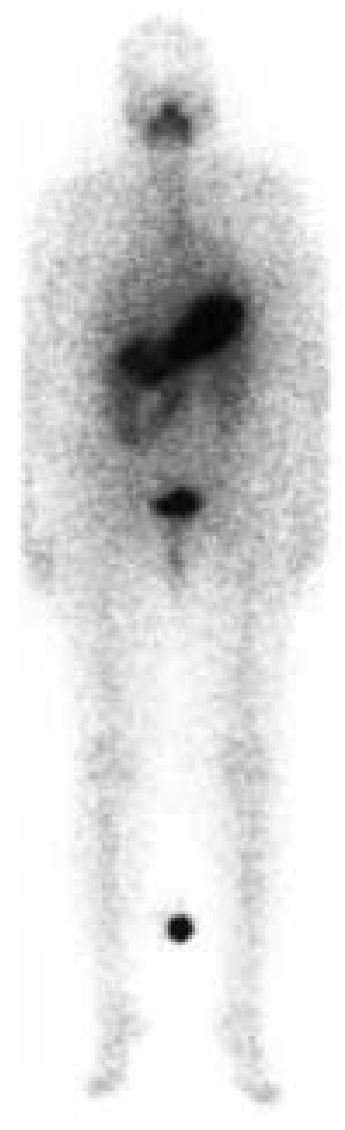

a) April 1999

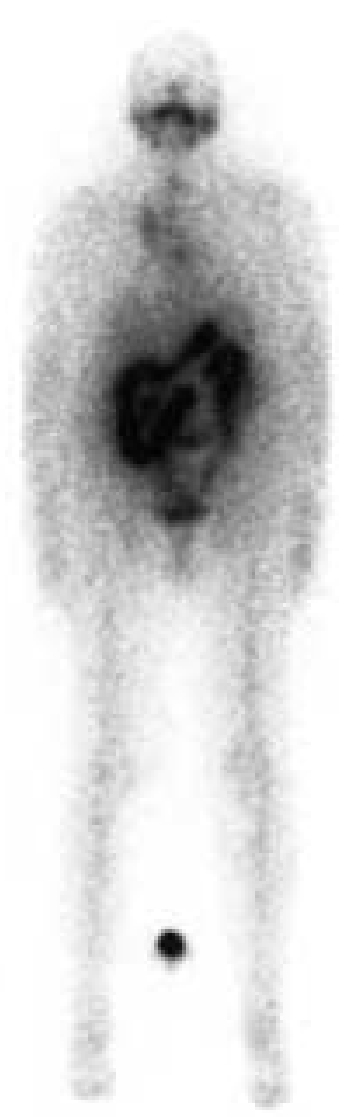

b) December 1999
Figure 1 Patient HS. In this patient a dedifferentiated papillary carcinoma had been incompletely resected at surgery. From the beginning there was evidence of lung metastases in the chest $\mathrm{X}$-ray. The patient underwent two radioiodine treatments, but no uptake could be seen in the lungs on post therapeutic scans (a). TG was only $2 \mathrm{ng} / \mathrm{ml}$. Both ${ }^{18} \mathrm{~F}-\mathrm{FDG}$ PET and CT scans showed extensive mediastinal and lung metastases, which were histologically proven. The patient was given isotretinoin $60 \mathrm{mg} / \mathrm{day}$ $(0.88 \mathrm{mg} / \mathrm{kg})$ for 91 days. The post therapeutic scan following isotretinoin pretreatment (b) showed only slightly increased uptake in the mediastinum and no uptake in the lung metastases. TG was $27 \mathrm{ng} / \mathrm{ml}$. Because of a clinically progressive disease the patient was referred for chemotherapy. She died 3 years after the initial diagnosis had been made.

In summary, out of five patients who were regarded as responders to isotretinoin treatment, two are completely free of symptoms, one is clinically relatively well, one has deteriorated, and one has died.

Side effects included (number of patients affected/ grading according to the Common Toxicity Criteria of the European Organisation for the Treatment of Cancer): a strong 'sunburn' (8/1-2), cheilitis (9/1-2), mucositis (5/1), conjunctivitis $(6 / 1)$, nose bleeds $(2 / 1)$, raised transaminases $(9 / 1-2)$, raised cholesterol $(2 / 1)$, raised creatinine $(2 / 1)$, anemia $(3 / 1)$, leukopenia
(4/1), and thrombopenia (2/1). In one further patient, whose data were not included, isotretinoin had to be stopped after 8 days due to nausea (grade 2 ) and vomiting (grade 2). Overall, $17 / 25$ patients (68\%) experienced clinical side effects, and $18 / 25$ patients $(72 \%)$ developed abnormal laboratory parameters. Side effects were of an overall tolerable level and could be controlled by patients keeping out of the sun as far as possible and applying moisturising cream to inflamed portions of their skin and to their lips. They were fully reversible within approximately 2 to 3 weeks after isotretinoin had been stopped.

\section{Discussion}

Isotretinoin was first used for induction of radioiodine uptake in patients with metastasized thyroid cancer who did not respond to radioiodine treatment in the late 1990s. A number of pilot studies reported encouraging results $(20-22)$.

Grünwald et al. (20) treated 12 patients with differentiated thyroid cancer who had a local recurrence or distant metastases untreatable by radioiodine, external radiation or surgery. Isotretinoin was given at $1-1.5 \mathrm{mg} / \mathrm{kg}$ body weight for 2 months prior to radioiodine treatment. They found a significant improvement in radioiodine uptake considered to be therapeutically useful in two patients. One of these had shown no uptake, and the other patient only a faint uptake previously. A faint improvement in uptake was seen in a further three patients who had previously exhibited no uptake. These five patients were considered responders. Levels of TG showed a marked increase in the responder group and were nearly unchanged in non-responders. Tumour size was not measured.

Simon et al. (21) treated 20 patients with differentiated thyroid carcinoma, including four oncocytic carcinomas. Patients had a locally invasive tumour or mediastinal or distant metastases that could not be treated with surgery or radioiodine. Retinoic acid was given at $1-1.5 \mathrm{mg} / \mathrm{kg}$ body weight for at least 5 weeks. Radioiodine uptake increased in eight of 16 patients who could be evaluated, five of which had previously been radioiodine-negative. Tumour size decreased in one of 15 patients who could be evaluated, increased in nine and remained unchanged in five patients. In contrast to the study of Grünwald et al. (20), which showed an increase in TG concentrations in responders, TG changes were indeterminate. They were not correlated to changes of radioiodine uptake, glucose metabolism or tumour size.

Pooled data from five centres in Germany, including those from the initial studies by Grünwald et al. (20) and Simon et al. (21), have been published (22). This report covered 50 patients who could be evaluated, but suffered from a high number of exclusions 


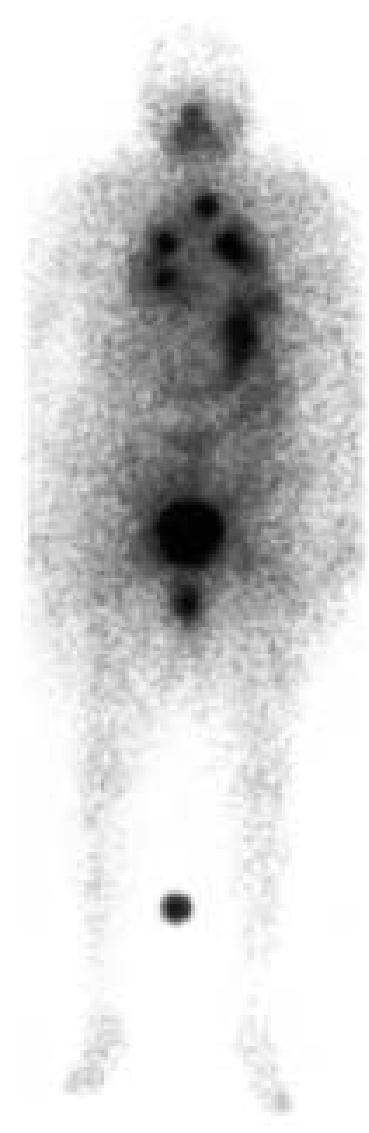

a) April 1997

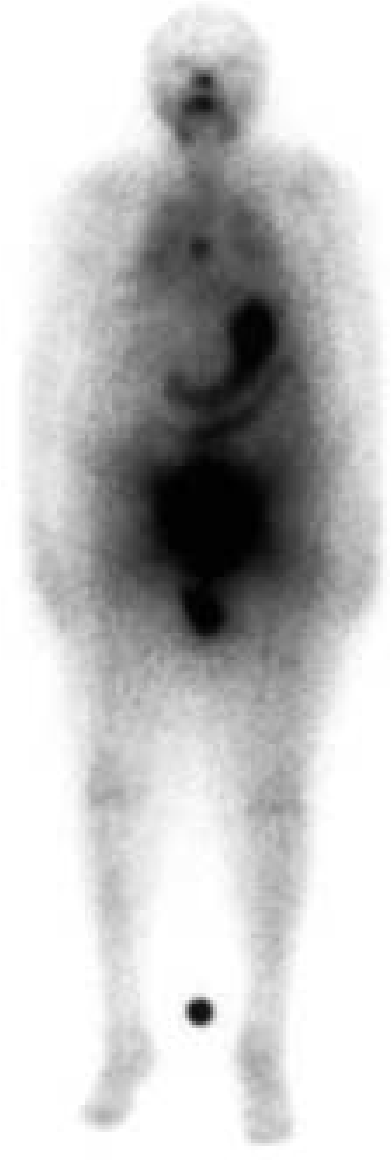

b) April 2000
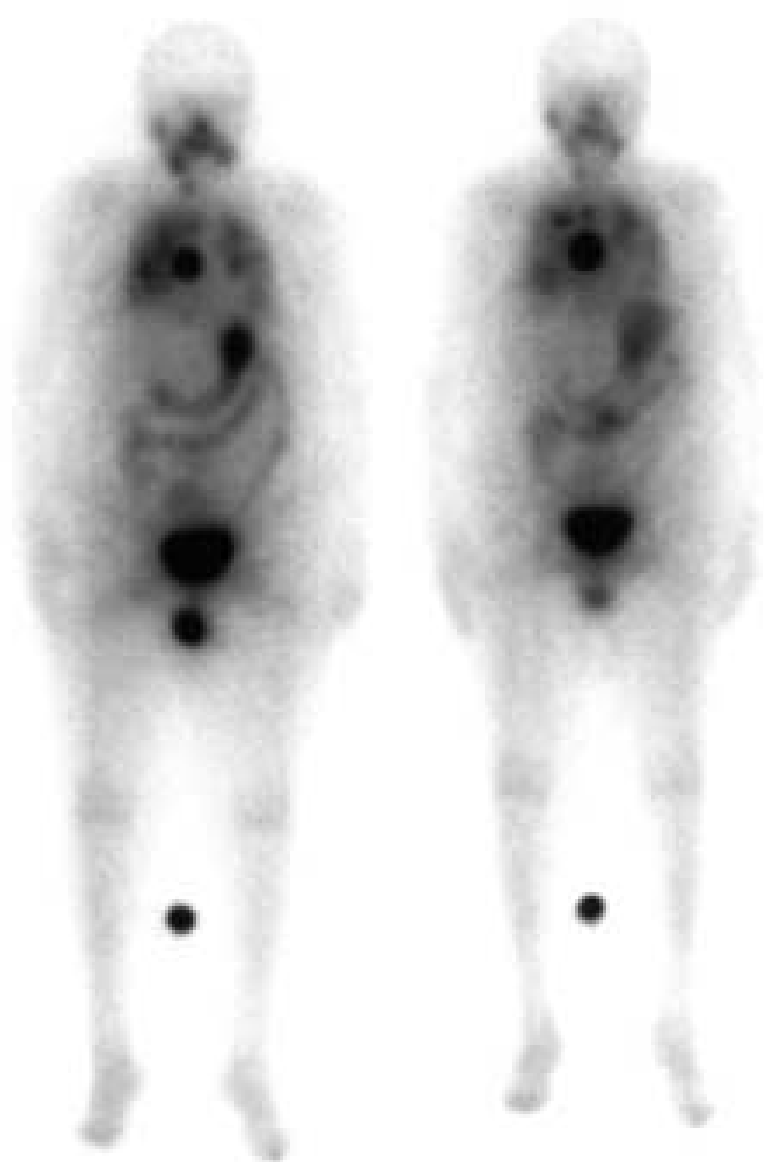

c) August 2000 d) January 2001

Figure 2 Patient AG. This 80-year-old woman with a follicular carcinoma underwent 11 radioiodine treatments from 1996 to 2001. Initially radioiodine scans showed intense uptake in both lungs and the upper mediastinum (a). After the ninth radioiodine therapy only reduced uptake was seen (b), whereas TG rose from $380 \mathrm{ng} / \mathrm{ml}$ to about $1100 \mathrm{ng} / \mathrm{ml}$. Therefore, progress of disease had to be assumed. The patient was given isotretinoin $60 \mathrm{mg} /$ day $(0.92 \mathrm{mg} / \mathrm{kg})$ for 90 days. The post therapeutic scan (c) showed clearly improved uptake in the lungs and mediastinum, and TG levels were $3300 \mathrm{ng} / \mathrm{ml}$. ${ }^{18} \mathrm{~F}-\mathrm{FDG}$ PET, chest X-ray and CT scan of the chest all failed to demonstrate metastatic disease. Isotretinoin pretreatment was repeated once with similar results (d). Since the patient was clinically asymptomatic, she elected not to have further treatment.

(one-third of patients initially enrolled were excluded), different protocols for the radioiodine scans (with regard to the activity administered, the method used to obtain TSH stimulation and the lack of quantitative imaging in the majority of patients), and the use of different TG assays. A strong point of this study is that tumour size could be measured by CT or magnetic resonance imaging (MRI) in three-quarters of patients. Radioiodine uptake increased in 21 patients, with a marked increase seen in 13 patients. TG increased in 30 patients, decreased in 12 patients, and remained unchanged in eight patients. Tumour size decreased in six patients, increased in nine patients, and remained unchanged in 22 patients. The overall clinical outcome defined (in declining order of importance) by tumour size, TG response and radioiodine uptake showed response to treatment in 10 patients, stable disease in nine patients, and disease progression in 31 patients. There was no clear correlation between radioiodine uptake and clinical outcome.

In our study, the number of patients who showed at least some improvement in radioiodine uptake was lower $(5 / 25=20 \%)$ than that reported by Grünwald et al. $(5 / 12=42 \%)$, Simon et al. $(8 / 16=50 \%)$ and in the German multicentre study $(21 / 50=42 \%)$. In contrast to the study of Grünwald et al. we could not observe any therapeutically relevant improvement of uptake in patients whose metastases were previously completely ${ }^{131}$ I-negative (group 1). In patients whose metastases showed at least some radioiodine uptake prior to isotretinoin treatment (group 2), a clearly improved uptake was seen in three out of 11 patients. 
For a number of reasons, it is difficult to distinguish an isotretinoin-induced change, if any, from the natural course of disease. Thyroid cancer is a slowly progressing malignancy. For a long period of time, this may even hold true in the minority of patients who experience some degree of dedifferentiation, commonly in the form of decreased radioiodine uptake, TG synthesis, or response to levothyroxine. Clinically evident progressive disease is usually only seen at a very late stage.

TG is not, in our opinion, a suitable parameter to indicate regressive/progressive disease. First, serum TG levels are dependent on TSH stimulation. The majority of patients treated with isotretinoin do not show an improved radioiodine uptake and thus will usually not be taken off levothyroxine for follow-up. TG measured at follow-up is, therefore, not comparable to measurements at the time of radioiodine treatment. Secondly, isotretinoin is known to induce TG mRNA (16). It may, therefore, be impossible to distinguish whether a raise in serum TG represents an effect of the drug or is due to progressive disease. On the other hand, a decrease in TG levels when the patient has been taken off isotretinoin may not necessarily be due to a response to treatment.

Radioiodine whole-body scans are more suitable to evaluate response to treatment, although the following should be taken into account: (i) radioiodine uptake is determined by a number of factors, including the degree of tumour dedifferentiation, TSH stimulation, diet, previous radioiodine treatments (stunning) and tumour mass. The cause of any change in uptake may, therefore, be difficult to determine. (ii) An improved radioiodine uptake does not in itself represent a benefit for the patient. (iii) An improved radioiodine uptake may occasionally be due to progressive disease rather than an effect of isotretinoin treatment. In patients with dedifferentiated thyroid cancer this would, however, not be a typical clinical course. (iv) Visual comparison of scans obtained on/off isotretinoin may sometimes, in our opinion, be misleading. Visual assessment is based (at least subconsciously) on a comparison of the uptake in a particular lesion with that of its surroundings, i. e. lesion contrast. An improved contrast may, therefore, be due to an improved uptake in the lesion or a decreased uptake in the background, which is affected by kidney function or state of hydration. ROI analysis which expresses lesion uptake as a percentage of the activity administered is, therefore, preferable. In this study, this effect may have occurred in patient RK who showed an improved uptake on visual assessment and improved contrast, but decreased uptake in ROI analysis. (v) Dose calculations have not been possible in this retrospective analysis, since radioiodine scans were only performed once so that biokinetic data are not available.

Boerner et al. (23) found that 3 months after initiation of isotretinoin treatment, the mean standardized uptake value (SUV) of ${ }^{18}$ F-FDG had decreased, and increased again once isotretinoin had been withdrawn. The decrease in SUV was more pronounced in patients showing improved radioiodine uptake after 6-9 months of isotretinoin treatment than in nonresponders, but there was no correlation between SUV and clinical outcome. The authors suggested that ${ }^{18}$ F-FDG PET may be used to obtain an early prediction of response to isotretinoin treatment. It should be noted, however, that the overlap in SUVs of responders and non-responders was large. Furthermore, Boerner et al. (23) used a fairly uncommon dosing scheme requiring isotretinoin administration at $0.3-$ $1.0 \mathrm{mg} / \mathrm{kg}$ body weight over a period of at least 9 months, without obtaining response rates higher than those seen when isotretinoin is given at a higher dose over a shorter period of time. Whilst there may be some clinical value in obtaining a prediction of response to treatment when isotretinoin is given longterm, this becomes less important if the drug is only given for 3 months.

From our study and the previous work of others, it may be concluded that isotretinoin gives some improvement of radioiodine uptake in metastases with low radioiodine uptake but does not appear to induce uptake in ${ }^{131}$ I-negative metastases. This is consistent with the finding in vitro that alkaline phosphatase, a differentiation marker, could be induced by isotretinoin in well-differentiated thyroid cancer cell lines but not in less well differentiated ones (12).

In summary, whilst isotretinoin has been shown to possess a number of effects in vitro which are of potential therapeutic interest, these have so far failed to translate into a marked clinical effect. The response rate in this study was in the order of $20 \%$ which is disappointingly low and does not confirm more enthusiastic results from previous studies. From our clinical experience over a period of 2 years we conclude that the therapeutic effect of isotretinoin in thyroid cancer is less than previously reported. An indiscriminate use of isotretinoin in all patients with otherwise untreatable thyroid cancer cannot be recommended. Controlled clinical trials using objective criteria of response should be undertaken.

\section{References}

1 Filetti S, Bidart JM, Arturi F, Caillou B, Russo D \& Schlumberger M. Sodium/iodide symporter: a key transport system in thyroid cancer cell metabolism. European Journal of Endocrinology 1999 $141443-457$.

2 Schmutzler C \& Köhrle J. Retinoic acid redifferentiation therapy for thyroid cancer. Thyroid $200010393-406$.

3 Schmutzler C \& Köhrle J. Innovative strategies for the treatment of thyroid cancer. European Journal of Endocrinology $2000 \mathbf{1 4 3}$ 15-24.

4 Brown SK \& Shalita AR. Acne vulgaris. Lancet $19983 \mathbf{3 1}$ 1871-1876.

5 Fenaux P, Chomienne C \& Degos L. Treatment of acute promyelocytic leukaemia. Best Practice and Research. Clinical Haematology 200114 153-174. 
6 Randolph TR. Acute promyelocytic leukemia (AML-M3). Part 1: pathophysiology, clinical diagnosis, and differentiation therapy. Clinical Laboratory Science 200013 98-105.

7 Slack JL. The biology and treatment of acute progranulocytic leukemia. Current Opinion in Oncology 199911 9-13.

8 Tallman MS. Therapy of acute promyelocytic leukemia: all-trans retinoic acid and beyond. Leukemia 199812 (Suppl 1) S37-S40.

9 Altucci L \& Gronemeyer H. The promise of retinoids to fight against cancer. Nature Reviews Cancer 20011 181-193.

10 Schmutzler C, Brtko J, Winzer R, Jakobs TC, Meissner-Weigl J, Simon D et al. Functional retinoid and thyroid hormone receptors in human thyroid-carcinoma cell lines and tissues. International Journal of Cancer 199876 368-376.

11 Schmutzler C, Brtko J, Bienert K \& Köhrle J. Effects of retinoids and role of retinoic acid receptors in human thyroid carcinomas and cell lines derived therefrom. Experimental and Clinical Endocrinology and Diabetes $1996 \mathbf{1 0 4}$ (Suppl 14) 16-19.

12 Schreck R, Schnieders F, Schmutzler C \& Köhrle J. Retinoids stimulate type I iodothyronine $5^{\prime}$-deiodinase activity in human follicular thyroid carcinoma cell lines. Journal of Clinical Endocrinology and Metabolism $199479791-798$.

13 Köhrle J, Oertel M, Hoang-Vu C, Schnieders F \& Brabant G. Type I5'-deiodinase a marker for differentiated thyroid carcinoma? Experimental and Clinical Endocrinology 199310160.

14 Schmutzler C, Winzer R, Meissner-Weigl J \& Köhrle J. Retinoic acid increases sodium/iodide symporter mRNA levels in human thyroid cancer cell lines and suppresses expression of functional symporter in nontransformed FRTL-5 rat thyroid cells. Biochemical and Biophysical Research Communications 1997240 832-838.

15 Van Herle AJ, Agatep ML, Padua DN III, Totanes TL, Canlapan DV, Van Herle HM et al. Effects of 13-cis-retinoic acid on growth and differentiation of human follicular carcinoma cells (UCLA R0 82 W-1) in vitro. Journal of Clinical Endocrinology and Metabolism 1990 $71755-763$.
16 Kurebayashi J, Tanaka K, Otsuki T, Moriya T, Kunisue H, Uno M et al. All-trans-retinoic acid modulates expression levels of thyroglobulin and cytokines in a new human poorly differentiated papillary thyroid carcinoma cell line, KTC-1. Journal of Clinical Endocrinology and Metabolism 200085 2889-2896.

17 Bassi V, Vitale M, Feliciello A, De Riu S, Rossi G \& Fenzi G. Retinoic acid induces intercellular adhesion molecule-1 hyperexpression in human thyroid carcinoma cell lines. Journal of Clinical Endocrinology and Metabolism $1995 \mathbf{8 0} 1129-1135$.

18 Hoang-Vu C, Bull K, Schwarz I, Krause G, Schmutzler C, Aust G et al. Regulation of CD97 protein in thyroid carcinoma. Journal of Clinical Endocrinology and Metabolism 199984 1104-1109.

19 Wunderlich G, Zöphel K, Crook L, Smith S, Smith BR \& Franke WG. A high-sensitivity enzyme-linked immunosorbent assay for serum thyroglobulin. Thyroid $200111819-824$.

20 Grünwald F, Menzel C, Bender H, Palmedo H, Otte R, Fimmers R et al. Redifferentiation therapy-induced radioiodine uptake in thyroid cancer. Journal of Nuclear Medicine $1998391903-1906$.

21 Simon D, Köhrle J, Reiners C, Börner AR, Schmutzler C, Mainz K et al. Redifferentiation therapy with retinoids: therapeutic option for advanced follicular and papillary thyroid carcinoma. World Journal of Surgery 199822 569-574.

22 Simon D, Körber C, Krausch M, Segering J, Groth P, Görges R et al. Clinical impact of retinoids in redifferentiation therapy of advanced thyroid cancer: final results of a pilot study. European Journal of Nuclear Medicine 200229 775-782.

23 Boerner AR, Petrich T, Weckesser E, Fricke H, Hofmann M, Otto D et al. Monitoring isotretinoin therapy in thyroid cancer using ${ }^{18} \mathrm{~F}$ FDG PET. European Journal of Nuclear Medicine 200229 231-236.

Received 26 September 2002

Accepted 21 January 2003 